\title{
Drug Use Evaluation of Ceftriaxone in Ras-Desta Memorial General Hospital, Ethiopia
}

This article was published in the following Dove Press journal:

Drug, Healthcare and Patient Safety

\section{Oumer Sada Muhammed (ID Beshir Bedru Nasir}

Department of Pharmacology and Clinical Pharmacy, School of Pharmacy, College of Health Sciences, Addis Ababa University, Addis Ababa, Ethiopia
Correspondence: Beshir Bedru Nasir Department of Pharmacology and Clinical Pharmacy,

School of Pharmacy, College of Health Sciences, Addis Ababa University, Churchill Avenue, P.O. Box II76, Addis Ababa, Ethiopia

Email beshir.bedru@aau.edu.et
Background: Ceftriaxone is one of the most commonly used antibiotics due to its availability, wide spectrum of activity and low toxicity. However, irrational use of ceftriaxone is one of the current issues in most countries, especially developing ones.

Objective: The aim of this study was to evaluate ceftriaxone utilization in different wards of Ras-Desta Memorial General Hospital (RDMGH) in Ethiopia.

Methods: An institution-based retrospective cross-sectional study was conducted on randomly selected 1,079 patients who were admitted to RDMGH from May 2017 to April 2018. A structured data abstraction format was used to collect data from patients' medical chart. Micromedex ${ }^{\circledR}$ drug interaction checker was used to identify significant drug-drug interactions, and national and international guidelines were used to evaluate the appropriateness of ceftriaxone use. The data were analyzed by using SPSS version 20.0.

Results: Among the 1,079 patients enrolled, ceftriaxone was utilized by $601(55.7 \%)$ patients, and these patients were considered for evaluation of appropriateness and subsequent analysis. Among 601 patients, ceftriaxone was used for therapeutic purpose in $362(60.2 \%)$ patients; of which, $359(99.2 \%)$ were for empiric therapy and the rest $239(39.8 \%)$ were for prophylaxis. In the majority of the patients, the dose of ceftriaxone was $2 \mathrm{~g} /$ day $472(78.6 \%)$ and for a duration of 2-7 days 409 (68.1\%). Inappropriate use of ceftriaxone was observed among 237 (39.4\%) patients with regard to indication, dose/frequency and duration. About half $(49.3 \%)$ of the deviation from the guidelines was observed from surgical and gynecologic/obstetrics wards. Among the co-prescribed medications with ceftriaxone, ringer lactate, warfarin and heparin were found to have a significant drug-drug interaction.

Conclusion: This study revealed that inappropriate use of ceftriaxone was high in RDMGH. This may increase the emergence of resistant pathogens which may lead to treatment failure and increase cost of therapy. Therefore, adherence to current evidence-based guidelines is recommended.

Keywords: antimicrobials, ceftriaxone, drug use evaluation; DUE, Ethiopia

\section{Introduction}

Antimicrobials have played a remarkable role in public health through decreasing of morbidity and mortality. ${ }^{1}$ The 19th and 20th centuries were peculiar in that health and longevity of populations progressed as the result of substantial achievements in controlling infectious diseases. This however, was not without challenges. ${ }^{2}$

The wide spread of misuse together with the emergence of antimicrobial resistance and escalating unwanted expenditures have resulted in antimicrobials being the drug most frequently chosen for drug use evaluation (DUE) studies. ${ }^{3}$ Ceftriaxone, as the most frequently used drug among the third generations cephalosporin class with reputable broad spectrum activity and utilization in many 
developing countries, ${ }^{4}$ was also highly prone to such problems. Studies reported that ceftriaxone was most often prescribed before culture and sensitivity tests and accurate decision was made in the diagnosis process. ${ }^{5}$

A high rate of inappropriate use of ceftriaxone $(87.9 \%)$ and impacts of antimicrobial resistance was documented in the world including the developing countries like Ethiopia. $^{5} \mathrm{~A}$ high rate of ceftriaxone resistance among gram negative bacteria was also reported across the European Union member states, which resulted in extra numbers of cases of infection, deaths, and hospital days. ${ }^{3}$

Inappropriate indication, empiric or prophylactic therapy and multiple drug uses were associated with misuse of antibiotics $^{6}$ and findings in German indicated a dramatic 10 fold increase in $E$. coli resistance to $3 \mathrm{rd}$ generation cephalosporin including ceftriaxone. ${ }^{7}$ Ceftriaxone resistance was also reported among E.coli (86.1\%) and K. pneumonia (95.8\%) in Sudanese general hospital. ${ }^{8}$

In Ethiopia, though there were no comprehensive nationwide studies to demonstrate the magnitude and direction of the problem, a few fragmented studies have documented the threatening incidences of inappropriate use of ceftriaxone. It was reported that there was an increasing rate of ceftriaxone utilization with a high rate of inappropriate use with regard to indication, presence of drug interaction, absence of lab investigation, duration of treatment and dosage regimen. ${ }^{5,9,10}$ This in turn was also associated with fueling an ever-increasing need for a newer antibiotics. Therefore, prudent prescribing of antimicrobial drugs is essential as it may reduce incidences of antimicrobial drug resistance. ${ }^{11}$

The literature review showed that the previous findings of ceftriaxone utilization in Ethiopia were a small sample size, in limited clinical settings, and evaluated the appropriateness against the Ethiopian standard treatment guideline developed for general hospitals. So, the use of a more rigorous evaluation tool, displaying the magnitude of the problem in a large sample size involving all clinical departments in a facility level is more reliable. Therefore, the objective of this study was to evaluate ceftriaxone utilization in different wards of Ras-Desta Memorial General Hospital (RDMGH), Addis Ababa, Ethiopia.

\section{Materials and Methods}

\section{Study Setting}

The study was conducted in RDMGH which is one of the state owned public hospitals in the capital city of Ethiopia.
The hospital provides a comprehensive medical service with its 550 staff, consisting of 246 medical staff and social workers, 98 technical and 206 administrative staff. The hospital has 168 beds in 5 inpatient wards (37 in gynecological and obstetric, 39 in surgical, 33 in medical, 19 in pediatric and 40 in adult and neonatal intensive care unit [ICU] wards).

\section{Study Design}

An institution-based retrospective cross sectional study design was employed to evaluate ceftriaxone utilization by reviewing medical records of patients who received ceftriaxone during May 1, 2017 to April 30, 2018. The DUE was made against the criteria of the currently developed protocol regarding the rational use of ceftriaxone. The treatment protocol was prepared by compiling current evidence-based recommendations regarding the use of ceftriaxone from WHO guidelines, Ethiopian standard treatment guideline for general hospital (STG 2014) ${ }^{12}$ and Infectious Disease Society of America (IDSA). STG 2014, which is a comprehensive guideline, used in Ethiopian general hospitals for the management of majority of diseases including surgical prophylaxis was extensively used for the protocol preparation. WHO guidelines (classification and management of childhood pneumonia ${ }^{13}$ and prevention of surgical site infection ${ }^{14}$ and IDSA practice guideline 2017 that includes several documents like management of infective endocarditis, meningitis and sepsis were also used. Micromedex ${ }^{\circledR}$ (Micromedex 2.0). Truven Health Analytics Inc) drug interaction checker was used to identify drug interactions. It is online application that provides evidence-based medical information including drug-drug interaction.

\section{Source and Study Population}

All patients admitted to RDMGH constituted the source population. The study population was all patients admitted to RDMGH during the study period and prescribed with at least 1 dose of ceftriaxone.

\section{Sample Size Determination and Sampling Procedure}

Because 385 is a minimum samples which is commonly considered using single population proportion formula with a proportion of $50 \%$ of inappropriate ceftriaxone utilization and confidence level of $95 \%$ with a $\mathrm{z}$-value of $1.96(\mathrm{P}=0.05) .{ }^{15}$ Large samples of 1,079 patients' medical charts were reviewed to get the assumed minimum 
samples that used ceftriaxone. However, among 1,079 medical charts reviewed 601 (55.7\%) were found to use ceftriaxone and considered for evaluation ceftriaxone use appropriateness and subsequent analysis. Systematic random sampling was used to employ the sample from a total of 3024 patients who were admitted during the data collection period (May 1, 2017 to April 30, 2018). Sampling fraction $(\mathrm{K})$ was calculated by dividing the total number of patients admitted in the study period $(3,024)$ by the estimated sample for the study (601) that results 5 . The first patients' medical chart was selected using simple random sampling technique then every 5 th chart was selected until the desired sample size was reached.

\section{Data Collection and Analysis}

Data was collected by 2 trained pharmacists through patients' medical chart review by using patient data abstraction tool. The content of the data collection tool was designed to record patient information, disease condition, working diagnosis, abnormal laboratory tests, abnormal diagnostic results, culture and sensitivity results, information regarding administration of ceftriaxone including its indication, dose, frequency of administration, duration of therapy, and information regarding coadministered medications. Five criteria, namely indication for use, dose, frequency of administration, duration of treatment, and drug interaction were used to evaluate appropriateness of ceftriaxone utilization based on the prepared protocol. After checking the completeness and appropriateness, the data was entered and analyzed by using Statistical Package for Social Science (SPSS) version 20.0. Descriptive statistics such as frequency distribution and percentages were performed to summarize the result. Mean with standard deviation was used to report continuous variables.

\section{Ethics Approval}

The study was approved by Department of Pharmacy, Faculty of Medical Sciences, Alkan Health Science, Business and Technology College (AHSBTC, ref 02/18). Confidentiality of the collected data was maintained throughout the study. Data was analyzed and reported in aggregate and all patient identifiers were avoided during data collection process.

\section{Results}

\section{Socio-Demographic Characteristics and Clinical Information of Patients}

Among the 1,079 patients enrolled, ceftriaxone was utilized in $601(55.7 \%)$ patients and these patients were considered for evaluation of appropriateness and subsequent analysis. The mean $\pm \mathrm{SD}$ age of the study participants was $35.0 \pm 17.7$ years ranging from 3 months to 89 years. The majority $(55.7 \%)$ of the patients were male and more than two thirds $(71.1 \%)$ did not have any chronic comorbidities. Among the study participants, 581 (96.7\%) had at least one type of routine investigation which was done before or during ceftriaxone therapy. Hematologic test $483(80.4 \%)$ was the most frequently conducted investigation followed by gram stain $66(11 \%)$.

Among 9 blood cultures tested, only 3 of them showed growth of a specific microorganism (E.coli). The remaining 6 patients whose culture did not show any microbial growth were treated empirically. Among 601 patients, ceftriaxone was used for therapeutic purpose in $362(60.2 \%)$ patients with (99.2\%) empiric therapy and the rest 239 (39.8\%) was used for prophylaxis. The prophylaxis uses were commonly for surgical prophylaxis but it was also used among a few patients who had soft tissue injuries.

The mean duration of ceftriaxone treatment was $5.2 \pm$ 3.8 days (ranging from stat to 50 days). Majority (68.1\%) of the patients were treated for 2-7 days and 18 (3\%) took ceftriaxone for more than 14 days. For the majority (472; $78.6 \%$ ) of the patients, the dose of ceftriaxone was $2 \mathrm{~g} /$ day (50 $\mathrm{mg} / \mathrm{kg} /$ day for pediatric age groups), followed by $2 \mathrm{~g}$ $4 \mathrm{~g} /$ day among 112 (18.6\%) patients (Table 1).

Maintenance fluid, diclofenac, and metronidazole were the top 3co-medications prescribed among 290 (48.2\%), 220 (36.6\%) and 74 (12.3\%) patients respectively (Table 2).

\section{Indication of Ceftriaxone Use and Compliance to National and International Guidelines}

Regarding compliance to guidelines on ceftriaxone utilization, $364(60.6 \%)$ of the samples were found to agree with the recommendations set in the guidelines. The rest (237; $39.4 \%$ ) of the ceftriaxone use was not consistent with the guidelines. Among the top 10 indications for which ceftriaxone was prescribed, pre-operative prophylaxis 239 (39.4\%) was the most common. Other indications for ceftriaxone use were pneumonia $(149 ; 24.5 \%)$, acute febrile illness (AFI) $(33 ; 5.4 \%)$ and urinary tract infection (UTI) $(30 ; 4.9 \%)$ (Table 3). Ceftriaxone was prescribed for AFI, AGE, wound, bronchial asthma, stroke and peptic ulcer disease which were not recommended by the guidelines as eligible indication. Noncompliance to guidelines 
Table I Socio-Demographic Characteristics and Clinical Information of Patients in RDMGH, June, 2018 ( $n=601)$

\begin{tabular}{|c|c|c|}
\hline Variables & & N (\%) \\
\hline Gender & $\begin{array}{l}\text { Male } \\
\text { Female }\end{array}$ & $\begin{array}{l}335(55.7) \\
266(44.3)\end{array}$ \\
\hline Age & $\begin{array}{l}3 \text { months to } 14 \text { years } \\
\text { I4-65 years } \\
\text { Above } 65 \text { years }\end{array}$ & $\begin{array}{l}40(6.7) \\
496(82.5) \\
65(10.8)\end{array}$ \\
\hline $\begin{array}{l}\text { Having chronic co- } \\
\text { morbidities }\end{array}$ & $\begin{array}{l}\text { Yes } \\
\text { No }\end{array}$ & $\begin{array}{l}427(71.1) \\
174(28.9)\end{array}$ \\
\hline $\begin{array}{l}\text { Routine investigation } \\
\text { conducted }\end{array}$ & $\begin{array}{l}\text { Hematologic tests } \\
\text { Urine analysis tests } \\
\text { Imaging } \\
\text { Widal weil felix tests } \\
\text { Gram stain } \\
\text { Blood culture }\end{array}$ & $\begin{array}{l}483(80.4) \\
405(67.4) \\
250(41.6) \\
161(26.8) \\
66(11.0) \\
9(1.5)\end{array}$ \\
\hline $\begin{array}{l}\text { Duration of } \\
\text { ceftriaxone therapy }\end{array}$ & $\begin{array}{l}\text { Single dose } \\
\text { One day ( } 2 \text { doses) } \\
2-7 \text { days } \\
8-14 \text { days } \\
>14 \text { days }\end{array}$ & $\begin{array}{l}31(5.1) \\
87(14.5) \\
409(68.1) \\
56(9.3) \\
18(3)\end{array}$ \\
\hline $\begin{array}{l}\text { Daily dose of } \\
\text { ceftriaxone utilized }\end{array}$ & $\begin{array}{l}2 \mathrm{~g} / \text { day }(50 \mathrm{mg} / \mathrm{kg} / \text { day for } \\
\text { pediatric age groups }) \\
2 \mathrm{~g}-4 \mathrm{~g} \\
4 \mathrm{~g}\end{array}$ & $\begin{array}{l}472(78.6) \\
112(18.6) \\
17(2.8)\end{array}$ \\
\hline $\begin{array}{l}\text { Reason for } \\
\text { prescribing ceftriaxone }\end{array}$ & $\begin{array}{l}\text { Therapeutic use } \\
\text { Prophylactic use }\end{array}$ & $\begin{array}{l}362(60.2) \\
239(39.8)\end{array}$ \\
\hline
\end{tabular}

was observed in $120(50.2 \%)$ of pre-operative uses; 3 $(20 \%)$ of sepsis; and $2(20 \%)$ of cellulitis treatment.

The highest proportion of ceftriaxone use was observed in internal medicine ward accounting for $290(48.3 \%)$ of the cases followed by $152(25.2 \%)$ in surgical, 91 (15.1\%) in gynecologic/obstetrics, $40(6.7 \%)$ in pediatrics and 28 (4.7\%) in emergency wards. Noncompliance to the guidelines was mainly identified from the emergency department $(19 ; 66.7 \%$ out of 28 cases), followed by surgical ward $(84 ; 55.6 \%)$, and gynecology/obstetrics ward (37; $40.7 \%$ ) (Figure 1). Compared to the internal medicine ward the rate of inappropriate ceftriaxone utilization was significantly higher in the emergency and surgery wards by 4.22 and 2.6 times (AOR=4.22, 95\% CI [1.80-12.24] and $\mathrm{AOR}=2.60,95 \% \mathrm{CI}[1.44-7.82]$ ), respectively.

About half (49.3\%) of noncompliance to the guidelines was found from surgical and gynecologic/obstetrics wards due to either longer duration of therapy or wrong timing of ceftriaxone use in surgical prophylaxis. Similarly, incorrect
Table 2 Top 10 Drugs Frequently Co-Prescribed with Ceftriaxone in RDMGH, June $2018(n=601)$

\begin{tabular}{|l|l|l|}
\hline Drugs & Frequency & Percent \\
\hline Maintenance fluids & 290 & 48.2 \\
Diclofenac & 220 & 36.6 \\
Metronidazole & 74 & 12.3 \\
Tramadol & 53 & 8.8 \\
Cimetidine & 46 & 7.7 \\
Paracetamol & $4 I$ & 6.8 \\
Gentamicin & 38 & 6.3 \\
Cloxacillin & 37 & 6.1 \\
Furosemide & 36 & 6.0 \\
Metoclopramide & 28 & 4.6 \\
\hline
\end{tabular}

indications and unacceptable duration of therapy for certain diagnosis composed the subsequent higher rate of the noncompliant cases $(88 ; 36.9 \%$ and $20 ; 7.6 \%)$. Regarding the general categories of inappropriate ceftriaxone use, unaccepted use for prophylaxis $(120 ; 50 \%)$ and wrongly indicated (87; 36.7\%) were the major problems identified in this study (Figure 2).

\section{Potential Drug-Drug Interaction Among Drugs Co-Prescribed with Ceftriaxone}

Among total of 46 drugs checked for potential drug-drug interactions with ceftriaxone, 4 drugs were shown to have amoderate interaction that needs monitoring. The drug interactions were identified among $54(9 \%)$ of the participants with ringer lactate 21 (3.5\%), doxycycline 16 $(2.7 \%)$, heparin $13(2.2 \%)$, and warfarin $4(0.7 \%)$.

\section{Discussion}

This study was designed to evaluate the appropriateness of ceftriaxone utilization in RDMGH. The current study revealed that a high rate $(55.7 \%)$ of ceftriaxone utilization was observed. Similar findings of high rate of ceftriaxone utilization (58\%) was reported in a study conducted in Tikur Anbessa specialized hospital, Ethiopia. ${ }^{5}$ High rate ceftriaxone utilization was also reported by other studies and the reasons might be attributed to its availability, effectiveness, broad spectrum and low toxicity rates. ${ }^{8}$ In Ethiopia ceftriaxone is the cheapest and commonly available parenteral antibiotics.

The mean duration of treatment in this study was 5.2 days (ranging from stat to 50 days) in fact more than two thirds $(68.1 \%)$ of the patients were treated for 2-7 days. The duration was lower than the findings reported from Ayder referral hospital 7.2 days $^{8}$ and Tikur Anbessa 
Table 3 Common Conditions for Which Ceftriaxone Was Prescribed and Respective Compliance to the Guidelines in RDMGH, June $2018(n=601)$

\begin{tabular}{|l|l|l|l|}
\hline \multirow{2}{*}{ Assessments } & Overall Use & \multicolumn{2}{|l|}{$\begin{array}{l}\text { Compliant to the } \\
\text { Guidelines }\end{array}$} \\
\cline { 2 - 4 } & N & $\mathbf{n}$ & $\%$ \\
\hline Pre-operative prophylaxis & 239 & 119 & 49.8 \\
Pneumonia & 149 & 133 & 89.3 \\
AFI & 33 & 0 & 0.0 \\
UTI & 30 & 29 & 96.7 \\
Meningitis & 19 & 17 & 89.5 \\
AGE & 18 & 0 & 0.0 \\
Sepsis & 16 & 13 & 81.3 \\
Bone \& STls & 11 & 10 & 90.9 \\
Cellulitis & 11 & 9 & 81.8 \\
UGIB/Ascites/Peritonitis & 11 & 10 & 90.9 \\
Wound & 11 & 0 & 0.0 \\
Endocarditis & 9 & 7 & 77.8 \\
Bronchial asthma & 7 & 0 & 0.0 \\
Stroke & 7 & 0 & 0.0 \\
Appendicular mass/Abscess & 6 & 6 & 100.0 \\
Other diseases & 24 & 16 & 66.7 \\
Total & $60 I$ & 364 & 60.4 \\
\hline
\end{tabular}

Abbreviations: AGE, acute gastroenteritis; AFI, acute febrile illness; UTI, urinary tract infection; STI, sexually transmitted infection; UGIB, upper gastrointestinal bleeding.

specialized hospital..$^{5}$ The shorter duration of therapy in this study might be due to this study included patients in surgery ward that needs short duration of therapy for prophylaxis and a more frequent step down switch of ceftriaxone (intravenous) to other (oral) antimicrobials. The median duration treatment in Victorian hospitals was also found to be only 3 days ${ }^{19}$ which is shorter than the median of the present study. The discrepancy could be attributed to difference in treated disease conditions and hospitals' protocols used to switch intravenous antibiotic to oral. Generally shorter duration of intravenous therapy would be desirable in most cases to reduce hospital stay and cost. ${ }^{20}$ However, severity and site of infection, numbers of comorbidities and patients' response to alternative oral regimens are important before making the decision.

In this study, the most common indications of ceftriaxone use were preoperative prophylaxis (39.8\%) and pneumonia $(24.8 \%)$. Similarly, preoperative prophylaxis and pneumonia were the common indication of ceftriaxone use in the findings of Ayder referral hospital ${ }^{10}$ and Dessie referral hospital. ${ }^{21}$

In this study, the most frequent daily dose of ceftriaxone was $2 \mathrm{~g}(78.6 \%)$ which is in line with the finding of Ayder referral hospital. ${ }^{10}$ However, a higher rate of dose of $2 \mathrm{~g} /$ day was reported from a study in Tikur Anbessa hospital $(88.9 \%) .^{5}$ The discrepancy could be due to the latter study did not include pediatric patients in which a lower daily dose of ceftriaxone was used. This dose was commonly used in Ethiopia for most cases and empiric therapy except for diseases that requires a higher dose, such as meningitis and infective endocarditis.

In this study, culture and sensitivity was done only for $9(0.8 \%)$ of the patients in which 3 of them showed bacterial growth and were sensitive to ceftriaxone. This is negligible as compared to studies conducted in India $(25.4 \%){ }^{2}$ Tikur Anbessa hospital, Ethiopia (22.4\%), Nepal (47.4\%), ${ }^{22}$ and Bangladesh (77\%). ${ }^{23}$ The reason of limited culture and sensitivity test in the current study might be due to lack of equipped microbiology laboratory and unaffordability among majority of the patients that

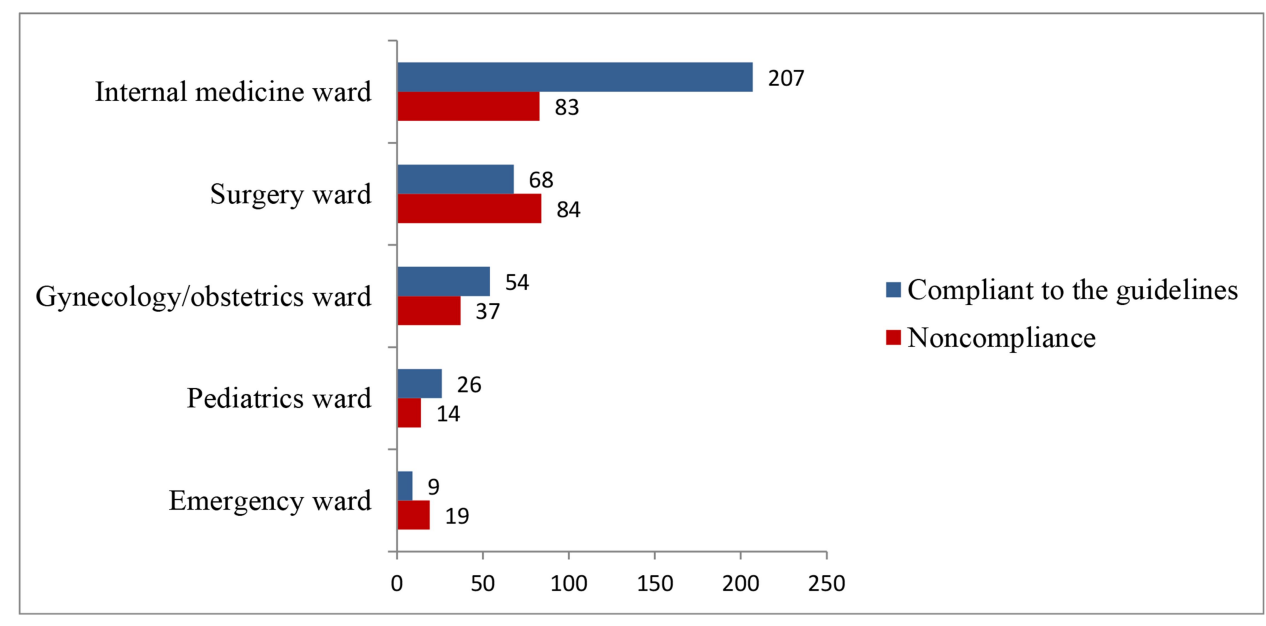

Figure I Compliance of ceftriaxone utilization to the guidelines in each department of RDMGH, June 2018 ( $n=60$ I). 


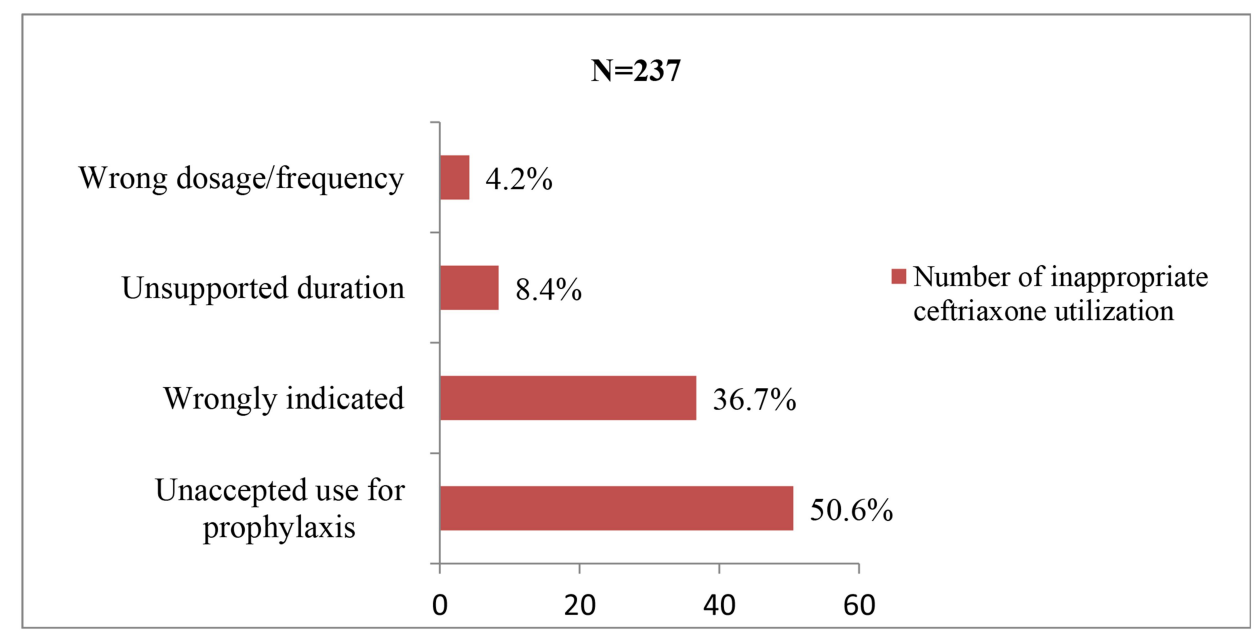

Figure 2 Categories inappropriate ceftriaxone utilization in RDMGH, June $2018(n=237)$.

leads to empiric therapy for almost all patients. However, a high rate of resistance to ceftriaxone was reported in Ethiopia in Tikur Anbessa specialized hospital $(62.5 \%)^{5}$ that needs due attention.

The inappropriate use of ceftriaxone in this study was found to be $39.6 \%$. This finding was with inline Dessie Referral Hospital $46.2 \%,{ }^{21}$ but lower than the findings of Tikur Anbessa specialized hospital (87.9\%), ${ }^{5}$ in Iran $(85.3 \%),{ }^{20}$ and Ayder Referral Hospital (64.2\%). ${ }^{10}$ The discrepancy of the findings might be attributed to a retrospective nature of the present study and the differences in the guidelines used to conduct the DUE. The other reason could be that, unlike this study, the above studies were conducted in referral hospitals in which treatment is complicated with several possible inappropriate medication uses. In addition, drug interaction in this study was not considered as inappropriate utilization that will lower the rate of inappropriate ceftriaxone utilization.

The highest proportion of ceftriaxone use 276 $(48.3 \%)$ was found in the medical wards followed by surgical wards $(25.2 \%)$. In contrary, inappropriate ceftriaxone use was frequently observed in emergency wards and the least in the medical ward. Compared to internal medicine wards a higher rate of inappropriate ceftriaxone utilization was observed in emergency and surgery wards by 4.22 and 2.6 times, respectively. Most of the deviations from the guidelines in the emergency ward were due to the overuse of antibiotics without optimal investigations and appropriate diagnosis. In addition, ceftriaxone was utilized for conditions without clear indications like patients diagnosed with AFI, wound, AGE, and bronchial asthma which were not supported in the guidelines. Surgical wards were the other areas with frequent inappropriate use of ceftriaxone for pre-operative prophylaxis. Similar finding was reported from the study of Ayder referral hospital. ${ }^{10}$ Though cefazolin is the recommended antibiotic preoperative prophylaxis for most surgeries, ceftriaxone was used for almost all surgery cases in the current study area. Furthermore, timing of initiation and longer duration of ceftriaxone prophylaxis was frequently observed in the present study.

Maintenance fluid, diclofenac, and metronidazole were the 3 commonly co-administered medication with ceftriaxone. Among drugs concomitantly administered with ceftriaxone, ringer lactate $(21 ; 3.5 \%)(16 ; 2.7 \%)$, heparin $(13$; $2.2 \%)$, and warfarin $(4 ; 0.7 \%)$ had a potential drug-drug interaction. Co-administration with heparin and warfarin may increase the risk of bleeding. Similar studies were reported in a study conducted in Tikur Anbessa specialized hospital. $^{5}$

\section{Limitation of the Study}

Despite the large sample size in most departments, it was a retrospective cross-sectional study that may under estimate the rate of inappropriate use of ceftriaxone. In addition, the evaluation was relied merely on the patients' medical records for which practices might have actually been different.

\section{Conclusion}

The majority (55.7\%) of patients admitted to RDMGH utilized ceftriaxone commonly for empiric therapy and prophylaxis purpose. Inappropriate use of ceftriaxone 
with regard to indication, dose/frequency and duration remains significant. This may increase the emergence of resistant pathogens which leads to treatment failure and increased cost of therapy. Therefore, adherence to current evidence-based guidelines is recommended. Few medications were also found to have a significant drug-drug interaction that requires a close monitoring or avoiding the concomitant administration.

\section{Abbreviations}

RDMGH, Ras-Desta Memorial General Hospital; AFI, acute febrile illness; UTI, urinary tract infection; STI, sexually transmitted infection; UGIB, upper gastrointestinal bleeding.

\section{Data Sharing Statement}

The datasets used and/or analyzed during the current study are available from the corresponding author on reasonable request.

\section{Acknowledgments}

We would like to express our deepest gratitude to all staff members of RDMGH for their unlimited support. We would also like to extend our sincere gratitude to the data collectors for their support throughout the study period and Dr. Abdurahman Ahmed for his support in language editing.

\section{Author Contributions}

Both authors made substantial contributions to conception and design, analysis and interpretation of data, took part in revising the article critically for important intellectual content, gave final approval of the version to be published and agrees to be accountable for all aspects of the work.

\section{Funding}

The authors received no specific funding for this work.

\section{Disclosure}

The authors report no conflicts of interest in this work.

\section{References}

1. Tulchinsky TH, Varavikova EA. What is the "new public health"? Public Health Rev. 2010;32(1):25. doi:10.1007/BF03391592

2. Hawkey P. The growing burden of antimicrobial resistance. J Antimicrob Chemother. 2008;62(suppl_1):i1-i9. doi:10.1093/jac/ dkn241

3. Mundy L, Pendry B, Rahman M. Antimicrobial resistance and synergy in herbal medicine. J Herb Med. 2016;6(2):53-58. doi:10.1016/j. hermed.2016.03.001
4. Al Akhali KM, Alzomar AK, Khan NA, Alavudeen SS. Misuse of antibiotics and awareness of antibiotic hazard among the public and medical professionals in Thamar province, in republic of Yemen. Pharm Glob. 2013;4(1):1.

5. Sileshi A, Tenna A, Feyissa M, Shibeshi W. Evaluation of ceftriaxone utilization in medical and emergency wards of Tikur Anbessa specialized hospital: a prospective cross-sectional study. $B M C$ Pharmacol Toxicol. 2016;17(1):7. doi:10.1186/s40360-016-0057-x

6. Pereira LMP, Phillips M, Ramlal H, Teemul K, Prabhakar P. Third generation cephalosporin use in a tertiary hospital in Port of Spain, Trinidad: need for an antibiotic policy. BMC Infect Dis. 2004;4(1):59. doi:10.1186/1471-2334-4-59

7. Meyer E, Schwab F, Schroeren-Boersch B, Gastmeier P. Dramatic increase of third-generation cephalosporin-resistant E. coli in German intensive care units: secular trends in antibiotic drug use and bacterial resistance, 2001 to 2008. Crit Care. 2010;14(3):R113. doi:10.1186/cc9062

8. Kheder SI. Cephalosporins usage and resistance trend in a Sudanese hospital surgical wards. J Pharma Biol Sci. 2011;11:11.

9. Shimels T, Bilal AI, Mulugeta A. Evaluation of ceftriaxone utilization in internal medicine wards of general hospitals in Addis Ababa, Ethiopia: a comparative retrospective study. $J$ Pharm Policy Pract. 2015;8(1):26. doi:10.1186/s40545-015-0047-1

10. Abebe FA, Berhe DF, Berhe AH, Hishe HZ, Akaleweld MA. Drug use evaluation of ceftriaxone: the case of Ayder Referral Hospital, Mekelle, Ethiopia. Int. J Pharm Sci Res. 2012;3(7):2191.

11. Gebretekle GB, Serbessa MK. Exploration of over the counter sales of antibiotics in community pharmacies of Addis Ababa, Ethiopia: pharmacy professionals' perspective. Antimicrob Resist Infect Control. 2016;5(1):2. doi:10.1186/s13756-016-0101-z

12. Eyasu M, Kiros K, Adamu Y, Tadesse Y, Keno A. Standard Treatment Guidelines for General Hospital. Addis Ababa, Ethiopa: Food Medicine and Healthcare Administration. 2014.

13. Organization WH. Revised WHO Classification and Treatment of Childhood Pneumonia at Health Facilities: Evidence Summaries. Geneva: World Health Organization; 2014.

14. Organization WH. Global Guidelines for the Prevention of Surgical Site Infection. World Health Organization; 2016.

15. Arifin WN. Introduction to sample size calculation. Educ Med J. 2013;5(2):2. doi:10.5959/eimj.v5i2.130

16. Jyothi K, Babu D. Drug utilization evaluation of Cephalosporins in general medicine units of rural tertiary care hospital. Int J Curr Pharm Res. 2012;4(2):88-91.

17. Chelkeba L. Antimicrobials use evaluation in Arba Minch Hospital, Gamo Gofa zone, South Ethiopia. Afr J Pharm. 2013;1(1):001-008.

18. Sapna P, Padma L, Veena D, Shanmukananda P. Drug utilization study of antimicrobials in post-operative wards in a teaching hospital. Int Res J Pharm App Sci. 2012;2(5):56-59.

19. Robertson MB, Dartnell JG, Korman TM, et al. Ceftriaxone and cefotaxime use in Victorian hospitals. Med J Aust. 2002;176 (11):524-529. doi:10.5694/j.1326-5377.2002.tb04549.x

20. Lee H, Jung D, Yeom JS, et al. Evaluation of ceftriaxone utilization at multicenter study. Korean J Intern Med. 2009;24(4):374. doi:10.3904/kjim.2009.24.4.374

21. Ayinalem GA, Gelaw BK, Belay AZ, Linjesa JL. Drug use evaluation of ceftriaxone in medical ward of Dessie Referral Hospital, North East Ethiopia. Int J Basic Clin Pharmacol. 2013;2(6):711-717. doi:10.5455/2319-2003.ijbcp20131208

22. Bajimaya S, Kansakar I, Sharma B, Byanju R. Outcome of cluster endophthalmitis in western plain region of Nepal. Kathmandu Univ Med J. 2010;8(1):102-108. doi:10.3126/kumj.v8i1.3232

23. Furqan S, Paracha SAU. Frequency of Streptococcus pneumonia and Haemophilus influenza in acute exacerbation of chronic obstructive airway disease and their sensitivity to levofloxacin. Age (Years). 2014;50(59):28.

24. Shohrati M, Hosseini S, Rahimian S, Parandeh AP. Assessment of reasonable use of ceftriaxone in internal and surgical wards. 2010. 


\section{Publish your work in this journal}

Drug, Healthcare and Patient Safety is an international, peer-reviewed open-access journal exploring patient safety issues in the healthcare continuum from diagnostic and screening interventions through to treatment, drug therapy and surgery. The journal is characterized by the rapid reporting of reviews, original research, clinical, epidemiological and post-marketing surveillance studies, risk management, health literacy and educational programs across all areas of healthcare delivery. The manuscript management system is completely online and includes a very quick and fair peer-review system. Visit http://www.dovepress.com/testimonials.php to read real quotes from published authors. 